\title{
EXTENDING POSITIVE DEFINITE LINEAR FORMS
}

\author{
JESÚS GIL DE LAMADRID
}

\begin{abstract}
In the classical literature two properties, called here symmetry and bounded variation, of a positive definite linear form $a^{\prime}$ on an involutive Banach algebra $\mathscr{A}$ are given. Together they form a necessary and sufficient condition that $a^{\prime}$ admit a positive definite linear extension to the involutive algebra obtained from $\mathscr{A}$ by adjoining an identity. In this note we show that bounded variation alone suffices, in that it already implies symmetry.
\end{abstract}

The properties in question are

$$
\left\langle a^{*}, a^{\prime}\right\rangle=\overline{\left\langle a, a^{\prime}\right\rangle} \quad \text { (symmetry) }
$$

and, for some $k, 0 \leqslant k<+\infty$,

$$
\left|\left\langle a, a^{\prime}\right\rangle\right|^{2} \leqslant k\left\langle a^{*} a, a^{\prime}\right\rangle \quad \text { (bounded variation), }
$$

for $a \in \mathscr{A}$. The term "bounded variation" is chosen because when $a^{\prime}$ (on a function algebra) is given by a measure, (2) boils down to the measure being finite.

We prove that (2) implies (1) by a bootstrap argument utilizing the classical result stating that both (1) and (2) do not only imply extensibility, but boundedness (continuity) of $a^{\prime}$ [2, p. 190]. Only this result is applied, not to $a^{\prime}$, but to an auxiliary form $a_{b}^{\prime}$, for which (1) and (2) are easily verified from the positive definiteness of $a^{\prime}$ alone, even if $a^{\prime}$ itself does not satisfy either condition. From this it follows that the Gelfand-Segal representation for $a^{\prime}$, which elsewhere requires (1) and (2) of $a^{\prime}$, can be constructed for positive definite $a^{\prime}$ without further assumptions. A weakened form

$$
\left\langle a^{*} b, a^{\prime}\right\rangle=\overline{\left\langle b^{*} a, a^{\prime}\right\rangle}
$$

of symmetry, (1), follows easily by polarization from positive definiteness; in particular from the fact that $\left\langle a^{*} a, a^{\prime}\right\rangle$ has only real values.

The Gelfand-Segal representation acts on a Hilbert space $H_{a^{\prime}}$ and there is a natural mapping $\eta: \mathscr{A} \rightarrow H_{a^{\prime}}$ with dense image such that

$$
(\eta a \mid \eta b)=\left\langle b^{*} a, a^{\prime}\right\rangle \text {. }
$$

Received by the editors May 23, 1983.

1980 Mathematics Subject Classification. Primary 46K99; Secondary 46K10.

Key words and phrases. Involutive Banach algebras, positive definite forms, symmetry, bounded variation, Gelfand-Segal representation, cyclic vectors, extensibility.

1984 American Mathematical Society $0002-9939 / 84 \$ 1.00+\$ .25$ per page 
That this, in fact, is an inner product on $\eta(\mathscr{A})$ follows from (3), and, of course, from positive definiteness of $a^{\prime}$. The representation is by operators $\pi(a)$, given on $\eta(\mathscr{A})$ by

$$
\pi(a) \eta b=\eta(a b)
$$

The auxiliary form $a_{b}^{\prime}$ is

$$
\left\langle a, a_{b}^{\prime}\right\rangle=\left\langle b^{*} a b, a^{\prime}\right\rangle .
$$

For $a_{b}^{\prime}$, which is obviously positive definite, (1) follows from (3) and (2) follows from the Schwarz inequality for $a^{\prime}$, a simple consequence of positive definiteness. This implies that $a_{b}^{\prime}$ is bounded, which, as shown in [2, p. 242], yields the fact that $\pi(a)$ is a bounded operator; hence, that $\pi$ itself is bounded. This shows that the GelfandSegal representation can always be constructed for $a^{\prime}$ on an involutive Banach algebra with or without identity, with no assumption on $a^{\prime}$ beyond positive definiteness.

Claim 1. Suppose $a^{\prime}$ is positive definite with bounded variation. Then there is a cyclic vector $h \in H_{a^{\prime}}$ such that

$$
\eta a=\pi(a) h
$$

and

$$
\left\langle a, a^{\prime}\right\rangle=(\pi(a) h \mid h)
$$

for every $a \in \mathscr{A}$.

Proof. We "transport" the linear form $a^{\prime}$ to $\eta(\mathscr{A})$, via

$$
\left\langle\eta a, h^{\prime}\right\rangle=\left\langle a, a^{\prime}\right\rangle \text {. }
$$

It follows from (2) that $h^{\prime}$ is both well defined (does not depend on the representative $a$ of $\eta a$ ) and bounded with respect to the Hilbert space norm of $\eta(\mathscr{A})$. Then, there is an $h \in H_{a}$, such that

$$
\left\langle a, a^{\prime}\right\rangle=(\eta a \mid h) .
$$

We establish (7) by "testing” it against an arbitrary $\eta b \in \eta(\mathscr{A})$. Thus

$$
(\eta b \mid \eta a)=\left\langle a^{*} b, a^{\prime}\right\rangle=\left(\eta\left(a^{*} b\right) \mid h\right)=\left(\pi\left(a^{*}\right) \eta b \mid h\right)=(\eta b \mid \pi(a) h),
$$

which establishes (7). Now (8) follows immediately from (10) and (7).

Claim 2. Every positive definite form of bounded variation on an involutive Banach algebra is symmetric; hence, bounded.

This is now immediate, because any $a^{\prime}$ given by (8) has all the desired properties.

\section{REFERENCES}

1. L. Loomis, An introduction to abstract harmonic analysis, Van Nostrand. New York, 1953.

2. M. A. Naimark, Normed rings, Noordhoff, Groningen, 1959.

Departement dF Mathematiques, Universite Paris VI, Paris, France

Department of Mathematics, University of Minnesota, Minneapolis, Minnesota 55455 